\title{
MiR-138-1-3p alters the stemness and radiosensitivity of tumor cells by targeting CRIPTO and the JAK2/STAT3 pathway in nasopharyngeal carcinoma
}

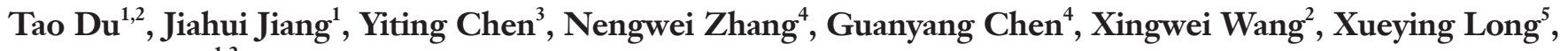 \\ Xueping Feng ${ }^{1,2 \wedge}$
}

${ }^{1}$ Institute of Medical Sciences, Xiangya Hospital, Central South University, Changsha, China; ${ }^{2}$ Department of Otolaryngology-Head and Neck Surgery, Xiangya Hospital, Central South University, Changsha, China; ${ }^{3}$ Department of Histology and Embryology, Xiangya School of Medicine, Central South University, Changsha, China; ${ }^{4}$ Department of General Surgery, Peking University Ninth School of Clinical Medicine, Beijing, China; ${ }^{5}$ Department of Radiology, Xiangya Hospital, Central South University, Changsha, China

Contributions: (I) Conception and design: X Feng, N Zhang; (II) Administrative support: X Wang; (III) Provision of study materials or patients: X Long; (IV) Collection and assembly of data: J Jiang, Y Chen; (V) Data analysis and interpretation: T Du, G Chen; (VI) Manuscript writing: All authors; (VII) Final approval of manuscript: All authors.

Correspondence to: Prof. Xueping Feng. Institute of Medical Sciences and Department of Otolaryngology-Head and Neck Surgery, Xiangya Hospital, Central South University, 87 Xiangya Road, Changsha 410008, China. Email: Xuepfeng@CSU.edu.cn; Dr. Xueying Long. Department of Radiology, Xiangya Hospital, Central South University, 87 Xiangya Road, Changsha 410008, China, Email: longxueying@CSU.edu.cn; Dr. Xingwei Wang. Department of Otolaryngology-Head and Neck Surgery, Xiangya Hospital, Central South University, 87 Xiangya Road, Changsha 410008, China. Email: wang-xingwei@126.com.

Background: Tumor resistance to radiotherapy is one of the main obstacles to the clinical treatment of nasopharyngeal carcinoma (NPC). Improving the radiosensitivity of tumor cells has an important clinical significance in treatment of clinical NPC. This study aimed to identify that miR-138-1-3p as a novel therapeutic target in radioresistant NPC cells and found its targets, CRIPTO and the JAK2/STAT3 pathway. Methods: Radioresistant C666-IR and HK-1R cells were derived from the NPC cell lines C666-1 and HK1. The different microRNAs (miRNAs) and their targeting genes were analyzed between C666-1 and C666IR cells using microarray bioinformatics. Western blot, qRT-PCR, gene transfection, Luciferase reporter assay, and confocal laser scanning microscopy were applied for the analysis of the different genes.

Results: MiR-138-1-3p was found to target CRIPTO, which involved in the epithelial-mesenchymal transition (EMT) and JAK2/STAT3 signaling pathways. The luciferase reporter assay confirmed that miR138-1-3p targeted CRIPTO and downregulated the expression of CRIPTO. Furthermore, miR-138-1$3 p$ affected the stability of the CRIPTO-GRP78 complex on the cell membrane and also reversed the radioresistant characteristics of NPC stem cells, which affected EMT and the JAK2/STAT3 signaling pathway. Conclusions: The miR-138-1-3p is a small molecule that can modulate radiosensitivity in the radioresistant C666-IR and HK-1R NPC cell lines by inhibiting EMT and targeting CRIPTO to reduce the activation of the JAK2/STAT3 pathway.

Keywords: MiR-138-1-3p; Nasopharyngeal carcinoma; epithelial-mesenchymal transition (EMT); Cripto; JAK2/ STAT3 pathway

Submitted Jan 14, 2021. Accepted for publication Mar 05, 2021.

doi: $10.21037 / \mathrm{atm}-21-521$

View this article at: http://dx.doi.org/10.21037/atm-21-521

^ ORCID: 0000000304813853. 


\section{Introduction}

Nasopharyngeal carcinoma (NPC) is a common malignancy of the head and neck region that is uncommon in the western world, but highly prevalent in Southeast Asia, particularly in south China (1). Its unique epidemiological distribution may be primarily attributed to Epstein-Barr virus infection, although other genetic and environmental factors play a contributing role. NPC is highly sensitive to ionizing radiation, therefore radiotherapy is the standard treatment to effectively control early tumors and achieve good prognosis (2). However, radioresistance is a serious problem where the radiation-resistant tumor cells are likely to remain and recur after radiotherapy, resulting in poor therapeutic outcome (3). Therefore, screening for early molecular biomarkers of radiotherapy resistance is important to improve the prognosis of NPC (4).

MicroRNAs (miRNAs) are a type of small non-coding RNA that can regulate gene expression by inhibiting mRNA translation or promoting mRNA degradation (5). Recent studies have shown that miRNAs can target key proteins in tumors after being affected by environmental factors, thereby promoting the development of cancer (6). Importantly, miRNAs can participate in cytochemical resistance or radiation resistance (7). For example, miR$193 a-3 p$ can modify the radioresistance and chemotherapy resistance of liver cancer cells by targeting PSEN1 (8). The interaction between miR-130a-3p and miR-17-5p can increase the radioresistance and chemoresistance of cardiac cancer cells (9). Therefore, miRNAs can be used as screening indicators to predict the efficacy of radiotherapy and chemotherapy (10). Several studies have reported the usefulness of miRNAs for the early detection of NPC (11). MiR-138-1-3p is a recently recognized miRNA, and its expression can affect the sensitivity of tumor cells to chemotherapy (12). MiR-138-1-3p is related to epigenetic mechanisms, transcription factor regulation, and hormone regulation (13). MiR-138-1-3p expression might be related the sensitivity of tumor cells to radiotherapy. It has not been reported whether miR-138-1-3p influence radiosensitivity of NPC. It is worth further study for the mechanism of miR-138-1-3p gene involved in NPC radiosensitivity.

The EGF-CFC (epidermal growth factor-Cripto/FRL1/ Cryptic with a cysteine-rich domain) family of proteins play an important role in the growth and development of vertebrates (14). Various studies have found that EGFCFC family proteins can promote tumor proliferation, invasion, and metastasis (15), and play a role in key signaling processes (16). CRIPTO is one such member of the EGFCFC co-receptor family (17), which is critical for inducing epithelial-mesenchymal transition (EMT) during embryonic development and in cancer. Increased expression of CRIPTO has been reported in various cancers, including breast, colon, lung, stomach, pancreatic, testicular, and ovarian cancer. CRIPTO overexpression has also been associated with the tumorigenesis and progression of NPC, potentially by inducing EMT of nasopharyngeal epithelial cells. The cancer stem cells arising from EMT are quiescent and apoptosisresistant, and have therefore been found to be radioresistant. Furthermore, the activation of EGF-CFC family proteins affects the JAK2/STAT3 signaling pathway (18), which has also been confirmed to be related to tumor progression (19). The phosphorylation of key proteins in the JAK2/STAT3 pathway can promote inflammation (20), while also contributing to radioresistance in cancer cells.

Cancer stem cells are a source of tumour recurrence in patients with NPC. The miR-138-1-3p might affect the sensitivity of NPC cells to radiation. MiR-138-1-3p might change the stem cells and radiosensitivity of NPC, and the miR-138-1-3p might be a clinical target in NPC radiotherapy. Therefore, the study is aimed to determine the role of miR-138-1-3p, which might be involved in the JAK2/STAT3 signaling pathway.

We present the following article in accordance with the MDAR reporting checklist (available at http://dx.doi. org/10.21037/atm-21-521).

\section{Methods}

\section{Cell culture}

The NPC cell lines C666-1 (human, XY-XB-3163) and HK-1 (human, SUER0025) were purchased from the Cell Bank of the Type Culture Collection of the Chinese Academy of Sciences (Shanghai, China). The radiation-resistant C666-1 cell line (C666-1R) was established by Professor Feng, Xiangya Hospital of Central South University, China. We then constructed the radiation-tolerant HK-1 cell line (HK-1R), as described below. All cells were cultured in RPMI-1640 medium (Biological Industries, 01-100-1A) containing $10 \%$ fetal bovine serum (FBS) (Biological Industries, $04-001-1 \mathrm{~A})$ at $37^{\circ} \mathrm{C}$ with $5 \% \mathrm{CO}_{2}$.

\section{Establishment of the radiation-resistant HK-1R cell line}

HK-1R cells were developed as described previously (21). 
Briefly, $1 \times 10^{6} \mathrm{HK}-1$ cells were inoculated into a $25 \mathrm{~cm}$ culture flask and cultured for 2 days in RPMI-1640 medium (Biological Industries, 01-100-1A) containing $10 \%$ FBS (Biological Industries, 04-001-1A) at $37^{\circ} \mathrm{C}$ with $5 \% \mathrm{CO}_{2}$. Then, cells were irradiated with $1 \mathrm{~Gy}$ of $\mathrm{X}$-rays by a high-energy linear accelerator, at a dose rate of 100 cGy/min (X-RAD 225 high-energy biological X-ray irradiator, USA). The medium was changed immediately after irradiation, and the cells were further incubated until $90 \%$ confluency was reached. Subsequently, the cells were collected after trypsinization and were subcultured into new flasks. Once these cells reached approximately $50 \%$ confluence, they were irradiated again with 100 cGy 3 times, 200 cGy 3 times, and 400 cGy 3 times. Thus, after treatment with a total dose of 2,100 cGy, we obtained the radiation-tolerant HK-1R cell population, which were cultured for at least 1 month before using in experiments.

\section{CCK-8 analysis}

The effect of irradiation on the viability of HK-1 and HK$1 \mathrm{R}$ cells was assessed using the CCK- 8 assay. The HK-1 and HK-1R cell suspensions were placed in a 96-well plate, and the plate was pre-cultured in an incubator for $24 \mathrm{~h}$, at $37{ }^{\circ} \mathrm{C}$ under $5 \% \mathrm{CO}_{2}$. The culture plate was irradiated with 600 cGy of X-rays through a high-energy linear accelerator (X-RAD 225 high-energy biological X-ray irradiator, USA) at a dose rate of $100 \mathrm{cGy} / \mathrm{min}$. After irradiation, the medium was changed immediately and cells were then incubated for $24 \mathrm{~h}$ under the same conditions as above. Then, we added $10 \mu \mathrm{L}$ of CCK-8 solution to each well, and further incubated the cells for $4 \mathrm{~h}$, after which the absorbance at $450 \mathrm{~nm}$ was measured with a multifunctional microplate reader (TECAN, Austria).

\section{Plate colony formation assay}

The HK-1 and HK-1R cells were irradiated with X-rays at specified doses of $0,2,4,6$, and 8 Gy. Dosimetry confirmed that the center dose rate was $100 \mathrm{cGy} / \mathrm{min}$. Subsequently, the irradiated cells and a set of unirradiated negative controls were incubated at $37{ }^{\circ} \mathrm{C}$ under $5 \% \mathrm{CO}_{2}$ humid air for 7 days. After washing 3 times with phosphatebuffered saline (PBS) (Beyotime Institute of Biotechnology, C0221A), the cells were fixed with methanol for $10 \mathrm{~min}$ and stained with $0.1 \%$ crystal violet (Beyotime Institute of Biotechnology, C0121) at $37^{\circ} \mathrm{C}$ for $10 \mathrm{~min}$.

\section{Microarray bybridization}

Total RNA from C666-1 and C666-1R cells, extracted using the TRIzol reagent, was quantified using the NanoDrop ND-2100 (Thermo Scientific, USA), and the RNA integrity was assessed using Agilent 2100 (Agilent Technologies, USA). The sample labeling, microarray hybridization, and washing was performed according to the manufacturer's instructions. Briefly, total RNA was tailed with poly A and labeled with biotin, after which the labeled RNA was hybridized onto the microarray. After washing, the slides were stained and the arrays were scanned by the Affymetrix Scanner 3000 (Affymetrix, USA).

\section{Data analysis}

Affymetrix GeneChip Command Console software (version 4.0 , Affymetrix) was used to analyze the array images and perform Robust Multi-Array Average (RMA) normalization. Next, Genespring software (version 12.5; Agilent Technologies, USA) was used for the resulting data analysis. Differentially expressed miRNAs were then identified through fold change, where a fold change of $>2$ was set as the threshold for up- and downregulated genes. Target genes of differentially expressed miRNAs were predicted with 3 databases: Targetscan, PITA, and microRNA.org. Gene Ontology (GO) and Kyoto Encyclopedia of Genes and Genomes (KEGG) analyses were applied to determine the roles of these target genes.

\section{Cell transfection}

Cell transfection was performed in HK1, HK-1R, and C666-1R cells using Lipofectamine 2000 (Invitrogen, 11668030). The miR-138-1-3p inhibitor and negative control (NC inhibitor) oligonucleotides, and miR-138-1-3p mimic and negative control (NC mimic) oligonucleotides were obtained from Sangon Biotech, Shanghai, China. After culturing the cells in a 6-well plate for $24 \mathrm{~h}$, the cells were appropriately transfected with the above-mentioned oligonucleotides, and after $48 \mathrm{~h}$ of transfection, the cells were collected and stored for later use.

\section{Quantitative real-time PCR (qRT-PCR)}

The relative expression of miR-138-1-3p in the transfected and naive HK-1, HK-1R, C666-1, and C666-1R cells was analyzed using qRT-PCR, following the manufacturer's instructions. 
Briefly, total RNA was extracted from the cells using the Trizol reagent (Invitrogen, A33250) and then reverse transcribed into cDNA using a reverse transcription kit (Transgen, AH341-01). Then, Top/Tip Green qPCR SuperMix (Transgen, AQ13101) was used for qRT-PCR analysis. The $2^{-\Delta \Delta \mathrm{Ct}}$ method was used to calculate the relative expression of miR-138-1-3p. The primer sequences were as follows: miR-138-1-3p, sense: 5'-GCUACUUCACAACACCAGGGCC-3'; antisense: 5'-GGCCCUGGUGUGUUGUGAAGUAGC. U6, forward primer, 5'-GCTTCGGCAGCACATATACTAAAAT-3'; reverse, 5'-CGCTTCACGAATTTGCGTGTCAT-3'.

\section{Transwell assay}

The Transwell assay was performed using a Costar Transwell culture plate (Corning Incorporated, USA) to evaluate the capability of cell migration in vitro. The transfected HK-1 and HK-1R cells $\left(5 \times 10^{4}\right.$ cells), suspended in serum-free medium, were placed in the upper chamber, while the $20 \%$ FBS-containing medium in the lower chamber served as the chemoattractant. After culturing for $36 \mathrm{~h}$, the cells that migrated to the lower chamber were fixed with methanol and stained with $0.1 \%$ crystal violet, and counted under an inverted microscope by picking 5 random fields of view.

\section{Wound healing assay}

Cell migration was further assessed using the wound healing assay. The transfected HK-1 and HK-1R cells were seeded and cultured in 6-well plates until $80 \%$ confluence. Then, the tip of a sterile $200 \mu \mathrm{L}$ pipette was used to manually create a wound between the cells. The cells were then washed and allowed to grow further in serum-free medium. Wound closure was observed every $2 \mathrm{~h}$ and imaged under a microscope after $36 \mathrm{~h}$.

\section{Western blot}

The Total Protein Extraction Kit (Transgen, DE10101) containing protease inhibitors was used to separate the total proteins of the NPC cells. Then, $10 \%$ sodium dodecyl sulfate polyacrylamide gel electrophoresis was used to separate an equal amount of cell protein, which was then transferred to a polyvinylidene fluoride membrane (EMD Millipore). The membrane was then blocked with $5 \%$ skimmed milk and incubated with the primary antibody at $4{ }^{\circ} \mathrm{C}$ overnight. After washing 3 times with Tris-buffered saline with Tween 20 (TBST buffer; Solarbio, T1081), the membrane was incubated with the secondary antibody for $2 \mathrm{~h}$ at $37^{\circ} \mathrm{C}$. Next, the samples were washed 3 times with TBST buffer (Solarbio, T1081), and the chemiluminescence gel imaging system (Bio-Rad, USA) was used to view protein bands. The antibodies used were as follows: GAPDH (Transgen, HC301-01), E-cadherin (Abcam, ab40772), N-cadherin (Abcam, ab18203), vimentin (Abcam, ab92547), CRIPTO (Abcam, ab108391), JAK2 (Abcam, ab108596), STAT3 (Abcam, ab68153), p-JAK2 (Abcam, ab32101), p-STAT3 (Abcam, ab76315), GRP78 (Abcam, ab21685), goat anti-mouse IgG (Transgen, HS201-01), and goat anti-rabbit IgG (Transgen, HS101-01).

\section{Luciferase reporter assay}

The relationship between CRIPTO and miR-138-1-3p was determined using the luciferase reporter assay. We amplified CRIPTO containing the predicted binding site (CRIPTOWT) or mutant binding site (CRIPTO-Mut) and cloned them into the pSI-Check2 vector (Hanbio, China). The miR-138-1-3p mimic, NC mimic, and plasmid were cotransfected into HEK-293T cells. At $48 \mathrm{~h}$ after transfection, the luciferase activity was measured using the dual luciferase reporter gene assay system (Promega, E1910).

\section{Immunobistochemistry}

The C666-1R cells were cultured on a glass slide, placed in a 6-well plate for $24 \mathrm{~h}$, and fixed with absolute ethanol for $30 \mathrm{~min}$. After washing with PBS, the non-specific binding sites were blocked with goat serum for $30 \mathrm{~min}$ at room temperature. After absorbing the goat serum using a filter paper, the cells were then incubated with the primary antibody overnight at $4{ }^{\circ} \mathrm{C}$. The next day, the cells were washed 3 times with PBS, blotted with a filter paper, and incubated with the secondary antibody at room temperature for $1 \mathrm{~h}$ in the dark. After washing with PBS, the nucleus was stained with DAPI (Solarbio, C0065) for $5 \mathrm{~min}$, and imaged using a confocal laser microscope (Leica, Germany).

\section{Statistical analysis}

Data were displayed as the mean \pm standard deviation. Differences were evaluated using a $t$-test. All statistical analyses were performed using GraphPad Prism 8. Statistical significance was defined as $\mathrm{P}<0.05$. 


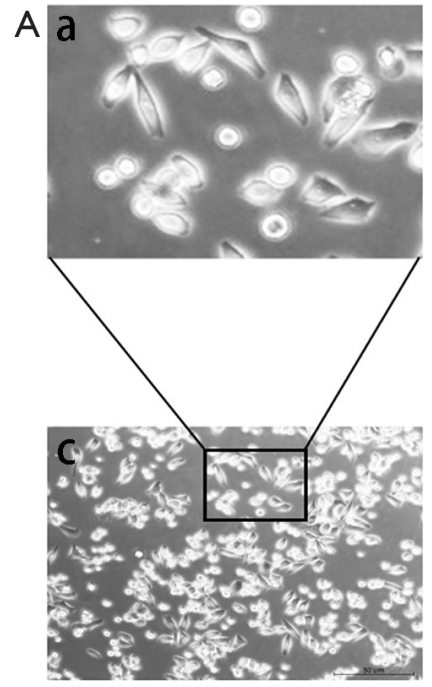

HK-1

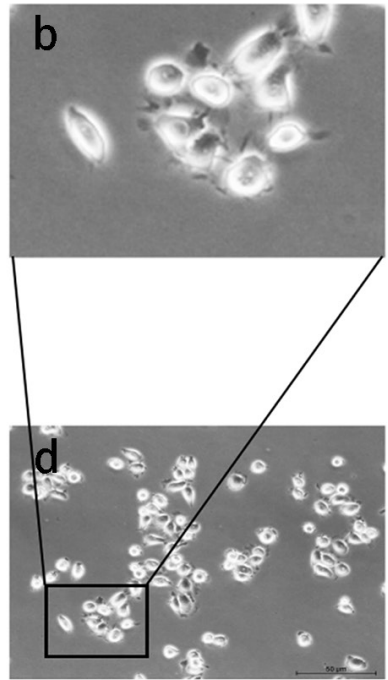

HK-1R

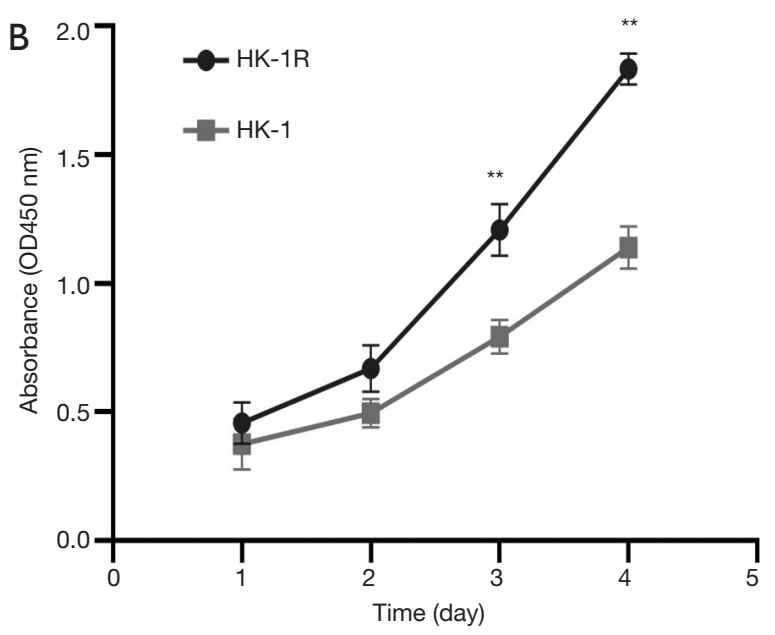

C 0 Gy 2 Gy 4 Gy 6 Gy 8 Gy
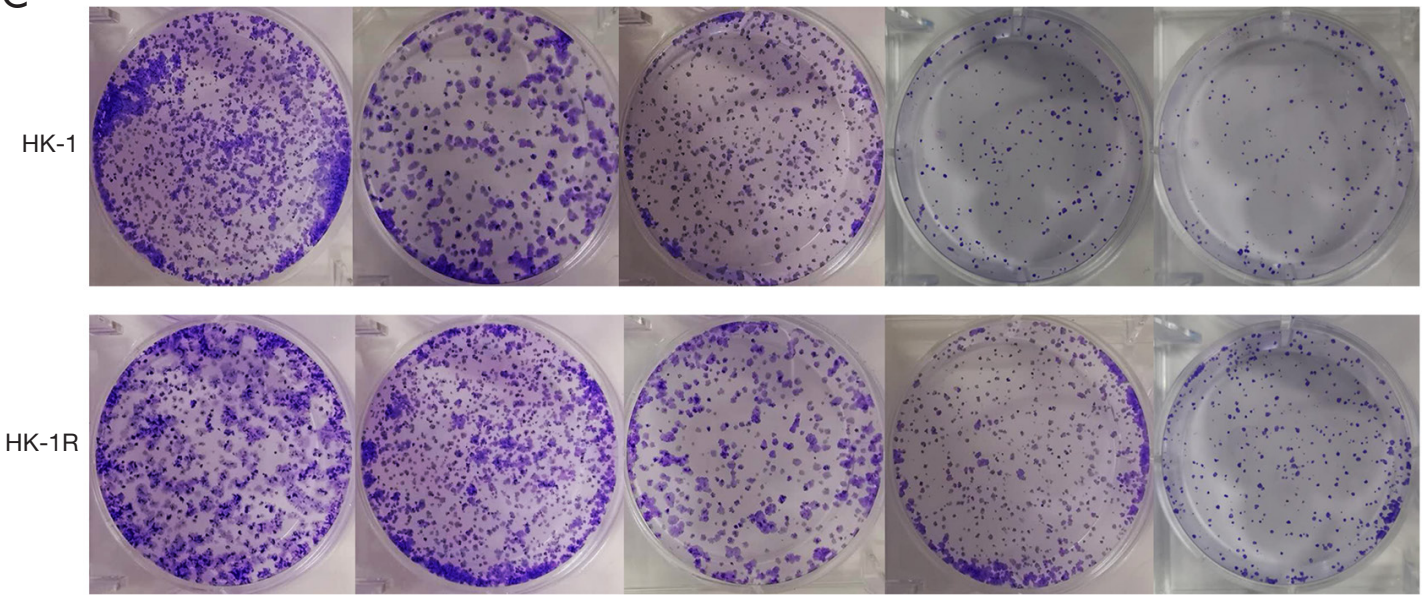

Figure $1 \mathrm{HK}-1 \mathrm{R}$ cells have stronger radiation resistance than HK-1 cells. (A) Under the microscope, the radiation resistant HK-1R cells were larger in size than the HK-1 cells, and displayed rounder morphology as compared to the elongated morphology of the parent cells: (a) and (b) are the enlarged images of the fixed field of view of (c) and (d). (B) Inoculating the same number of cells $\left(2 \times 10^{3}\right)$, the viability of HK-1 and HK-1R cells after 6 Gy irradiation was detected by the CCK-8 assay, and the cell proliferation activity of HK-1R cells was significantly higher than that of HK-1 cells. (C) Concurrently, HK-1R cells displayed stronger colony formation capability than HK-1 cells under the same radiation dose, representative images of colony formation of HK-1 (600 cells/well) and HK-1R cells (300 cells/well) exposed to $0,2,4,6,8$ Gy rays. ${ }^{* *} \mathrm{P}<0.01$.

\section{Results}

\section{Radiation-resistant HK-1 cell line}

We developed the radiation resistant version of HK-1 cells by irradiating them at different doses of $\mathrm{X}$-rays. Under the microscope, the HK-1R cells were larger in size than the HK-1 cells, and displayed rounder morphology as compared to the elongated morphology of the parent cells (Figure 1A). The cell proliferation activity of HK-1R cells after 6 Gy irradiation was significantly higher than that of HK-1 cells (Figure 1B). Concurrently, after X-ray irradiation, HK$1 \mathrm{R}$ cells displayed stronger colony formation capability than HK-1 cells under the same radiation dose (Figure 1C), indicating lower sensitivity of HK-1R cells to radiation than 
HK-1 cells.

\section{MiR-138-1-3p is related to the sensitivity of NPC cells to radiation}

Compared with its parent cell line, C666-1, C666-1R cells have a stronger capacity to resist radiation exposure. After analyzing the miRNA microarray results of these 2 cell lines, we found that miR-138-1-3p was significantly downregulated in C666-1R cells, by a factor of 13.49 (Figure 2A). Our qRT-PCR results also confirmed the microarray results, where the miR-1381-3p levels were significantly reduced in C666-1R cells, compared to C6661 cells $(\mathrm{P}<0.01$; Figure $2 B)$. Following this trend, qRTPCR analysis of HK-1 and HK-1R cells demonstrated significantly reduced miR-138-1-3p expression in the radiation-resistant cell line compared to its parent cell line $(\mathrm{P}<0.01 ;$ Figure 2C). Therefore, we speculated that the expression of miR-138-1-3p may be closely related to the radiosensitivity of NPC cells.

To specifically investigate the role of miR-138-1-3p in the development of radioresistance in NPC cells, we performed miR-138-1-3p transfection experiments in HK-1 and HK-1R cells. The qRT-PCR results confirmed that the expression of miR-138-1-3p was decreased in miR-138-1-3p-inhibitor transfected HK-1 cells, as compared to the $\mathrm{NC}$ inhibitor cells $(\mathrm{P}<0.01$; Figure $2 D)$. Additionally, miR-138-1-3p expression increased in miR138-1-3p-mimic transfected HK-1R cells, as compared to the NC mimic cells $(\mathrm{P}<0.01$; Figure $2 E)$. Subsequently, the results of the plate clone formation experiment demonstrated that the inhibition of miR-138-1-3p increased the radioresistance of HK-1 cells, while the overexpression of miR-138-1-3p increased the radiosensitivity of HK-1R cells (Figure 2F). These results indicate that miR-138-1-3p can alter the radiosensitivity of NPC cells.

\section{MiR-138-1-3p enbances the radiosensitivity of NPC cells by inbibiting EMT}

It has been reported that EMT is related to radiation resistance in cancer (22). Therefore, we speculated that miR-138-1-3p affects the radiosensitivity of NPC cells through EMT. Cell migration is the most distinctive feature of EMT (23). Transwell experiments showed that miR-1381-3p inhibition significantly increased HK-1 cell migration (NC inhibitor: $\mathrm{miR}-138-1-3 \mathrm{p}$ inhibitor $=1: 4, \mathrm{P}<0.05$; Figure $3 A$, a and b), while its overexpression significantly reduced the migration capability of $\mathrm{HK}-1 \mathrm{R}$ cells (NC mimic: miR-138-1-3p mimic $=3: 1, \mathrm{P}<0.05$; Figure $3 A$, c and $\mathrm{d}$ ). Correspondingly, wound healing experiments also demonstrated that miR-138-1-3p affects the migration of HK-1 cells (NC inhibitor: miR-138-1-3p inhibitor $=0.55: 0.24, \mathrm{P}<0.01 ;$ Figure $3 B$ ) and $\mathrm{HK}-1 \mathrm{R}$ cells $(\mathrm{NC}$ mimic: miR-138-1-3p mimic $=0.19: 0.52, \mathrm{P}<0.01$; Figure $3 B$ ). In addition, western blot experiments showed that after miR-138-1-3p inhibition, the epithelial marker E-cadherin was downregulated in HK-1 cells (Figure 3C), while interstitial markers such as $\mathrm{N}$-cadherin and vimentin were upregulated (Figure 3C). Similarly, after upregulating miR-138-1-3p in HK-1R cells, the protein expression of $\mathrm{E}$-cadherin, $\mathrm{N}$-cadherin, and vimentin were reversed, as expected (Figure 3C). Overall, these results confirm that miR-138-1-3p can reduce the radioresistance of NPC cells by inhibiting EMT.

\section{MiR-138-1-3p affects the radiosensitivity of NPC cells by targeting CRIPTO}

We attempted to find the downstream protein of miR-138-1-3p by using the Target Scan Human 7.2 database (http://www.targetscan.org/vert_72/) to investigate how it can modulate the sensitivity of NPC cells to ionizing radiation. Based on the results, we hypothesized that miR-138-1-3p can target the CRIPTO protein (Figure $4 A$ ), which is an external protein anchored on the cell membrane that interacts with the GRP78 protein. Their combination in the cell membrane can transform tumor cells into stem cells (24), and promote cell growth (25). Studies have shown that targeting GRP78 on tumor cells can effectively inhibit the stemness, radioresistance, and tumorigenicity of head and neck carcinomas (26). Our dual luciferase reporter assay proved that miR-138-1-3p and CRIPTO have targeted sites (Figure 4B). Furthermore, western blot analysis showed a significant change in the CRIPTO and GRP78 expression levels with the change in miR-138-1-3p level (Figure 4C). These results indicate that miR-138-1-3p can alter the radioresistance of NPC cells by regulating CRIPTO expression.

\section{MiR-138-1-3p affects the stability of the CRIPTO and GRP78 complex on the cell membrane}

We detected the expression of CRIPTO in HK-1, HK1R, C666-1, and C666-1R cells (Figure 5A) and selected C666-1R cells for miR-138-1-3p inhibitor and miR-138-1- 
A

ID
Accession
FC
C666-IR vs C666-1
SITES
SEQUENCE

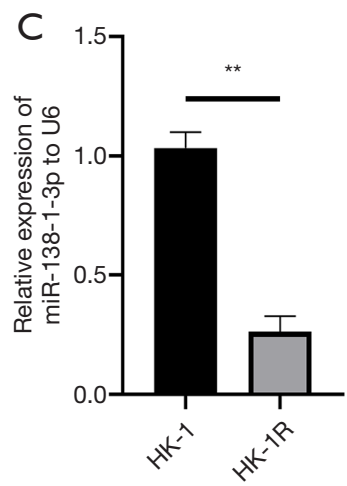

has-miR-138-1-3p

MIMAT0004607

$-13.49$

down chr3:44155766-44155787(+) GCUACUUCACAACACCAGGGCC
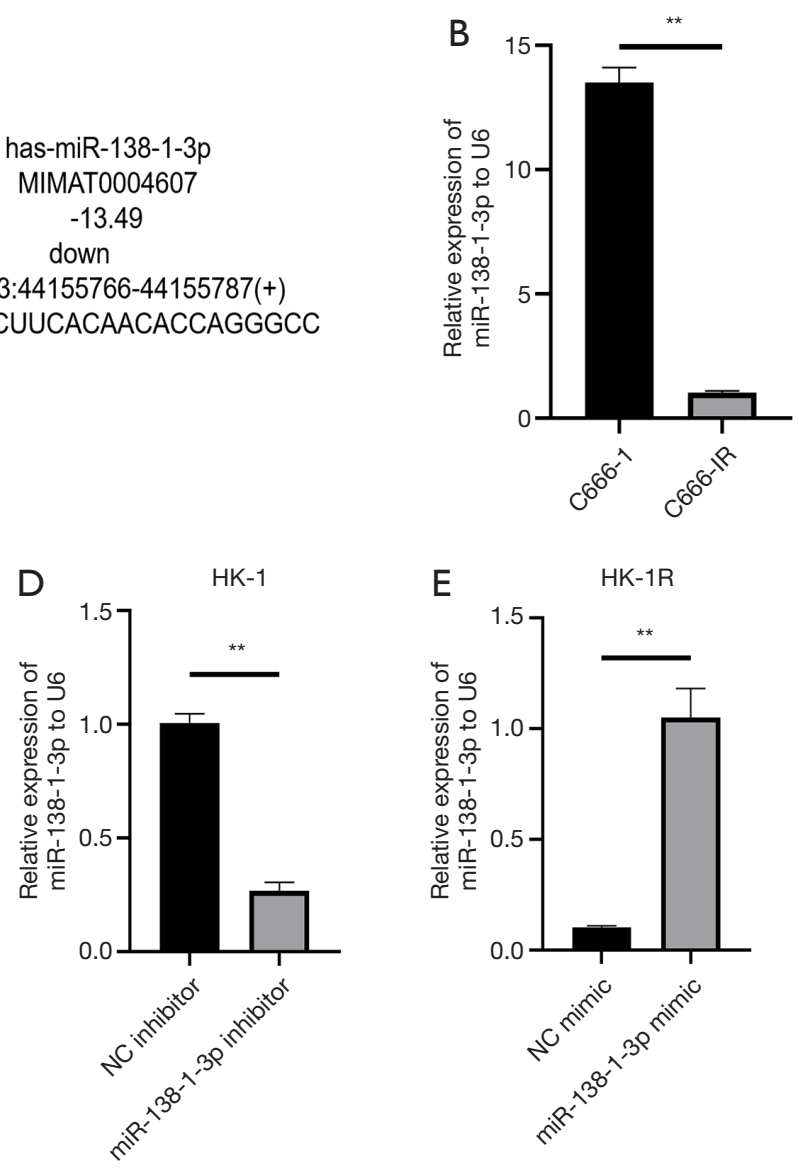

$\mathrm{F}$

HK-1

HK-1R

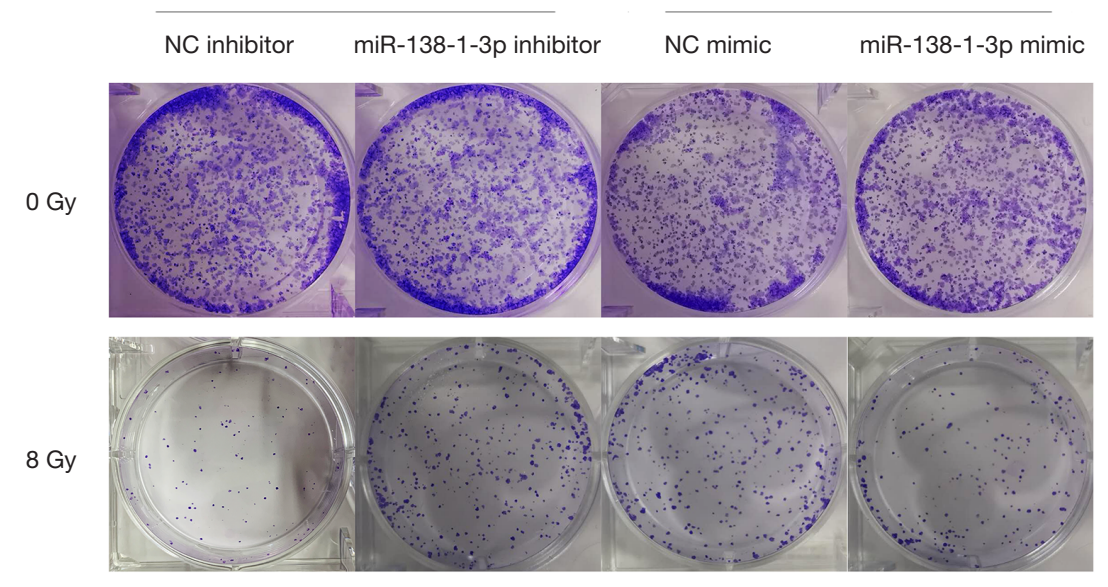

Figure 2 MiR-138-1-3p increases the radiosensitivity of nasopharyngeal carcinoma (NPC) cells. (A) Analysis of miRNA chip data of C6661 and C666-IR cells. (B) The relative expression of miR-138-1-3p in C666-1 and C666-IR cells was detected by qRT-PCR. (C) The relative expression of miR-138-1-3p in HK-1 and HK-1R cells was detected by qRT-PCR. (D) The expression of miR-138-1-3p after HK-1 cells were transfected with NC inhibitor and miR-138-1-3p inhibitor. (E) The expression of miR-138-1-3p after HK-1R cells were transfected with NC mimic and miR-138-1-3p mimic. (F) The plate cloning experiment results of HK-1 (600 cells/well) and HK-1R (300 cells/well) cells after 8 Gy irradiation were observed. ${ }^{* *} \mathrm{P}<0.01$. 
A

HK-1

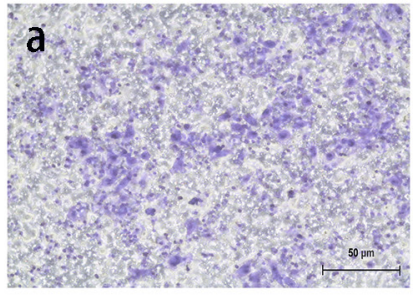

NC inhibitor

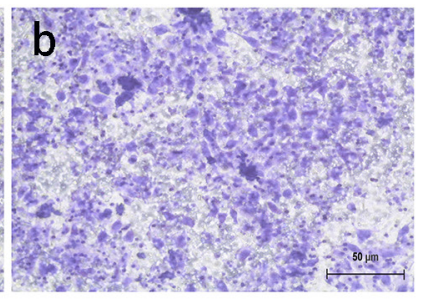

miR-138-1-3p inhibitor

HK-1R

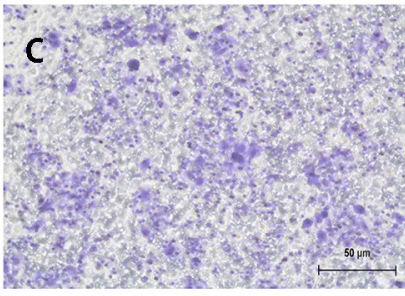

NC mimic

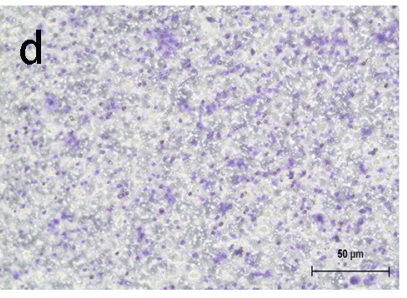

miR-138-1-3p mimic

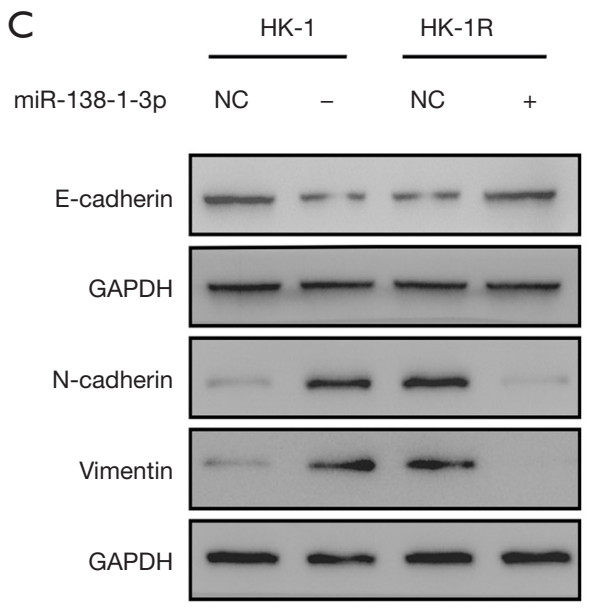

B

$\mathrm{HK}-1$
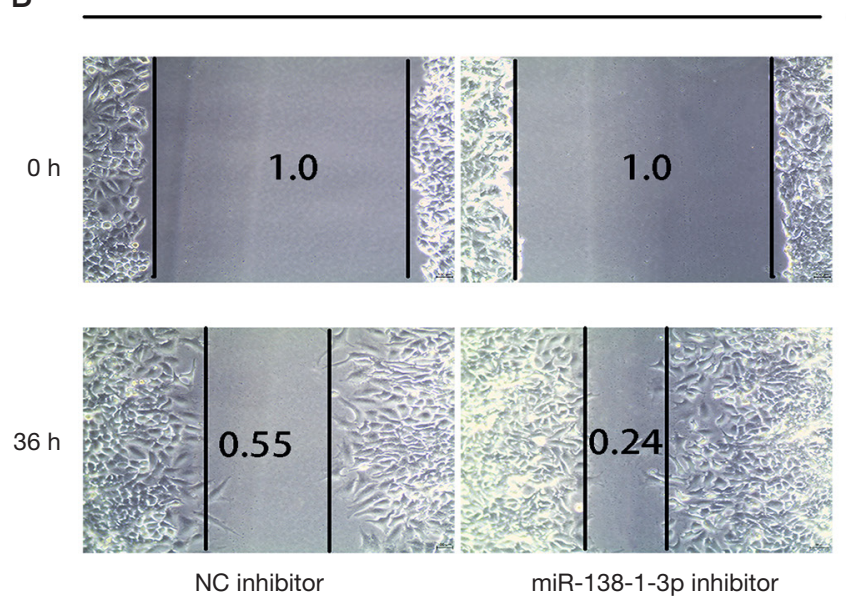

miR-138-1-3p inhibitor

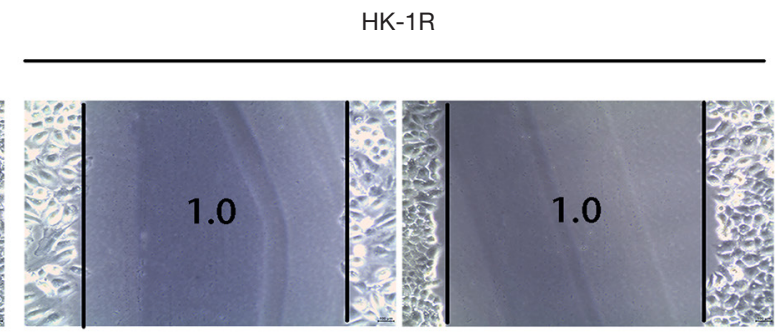

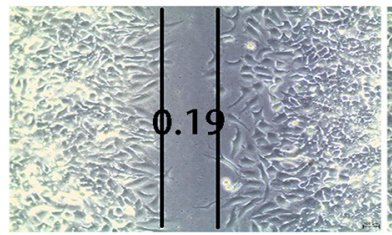

NC mimic

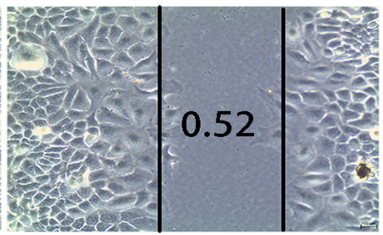

miR-138-1-3p mimic

Figure 3 Upregulated miR-138-1-3p inhibits epithelial-mesenchymal transition (EMT) of nasopharyngeal carcinoma (NPC) cells. (A) Transwell assay of HK-1 and HK-1R cells after altering miR-138-1-3p levels: HK-1 transfected with NC inhibitor (a); HK-1 transfected with miR-138-1-3p inhibitor (b); HK-1R transfected with NC mimic (c); HK-1R transfected with miR-138-1-3p mimic (d). (B) The migration ability of HK-1 and HK-1R cells detected by the wound healing assay after altering miR-138-1-3p levels. (C) The expressions of E-cadherin, N-cadherin, and vimentin in HK-1 and HK-1R cells after altering miR-138-1-3p levels.

$3 \mathrm{p}$ mimic transfection (Figure 5B). After immunostaining, the images from laser confocal microscopy revealed a significantly reduced colocalization signal (Figure 5C) of CRIPTO and GRP78 on the cell membrane after miR138-1-3p upregulation. Correspondingly, the greenlabeled CRIPTO signal was weakened (Figure 5C) and the CRIPTO-GRP78 complex was dominated by the redlabeled GRP78 signal (Figure 5D). Similarly, in response to downregulation of miR-138-1-3p, the colocalization signal of CRIPTO and GRP78 was enhanced, as the green-labeled CRIPTO signal was significantly enhanced (Figure 5C), and the CRIPTO-GRP78 complex was dominated by the 


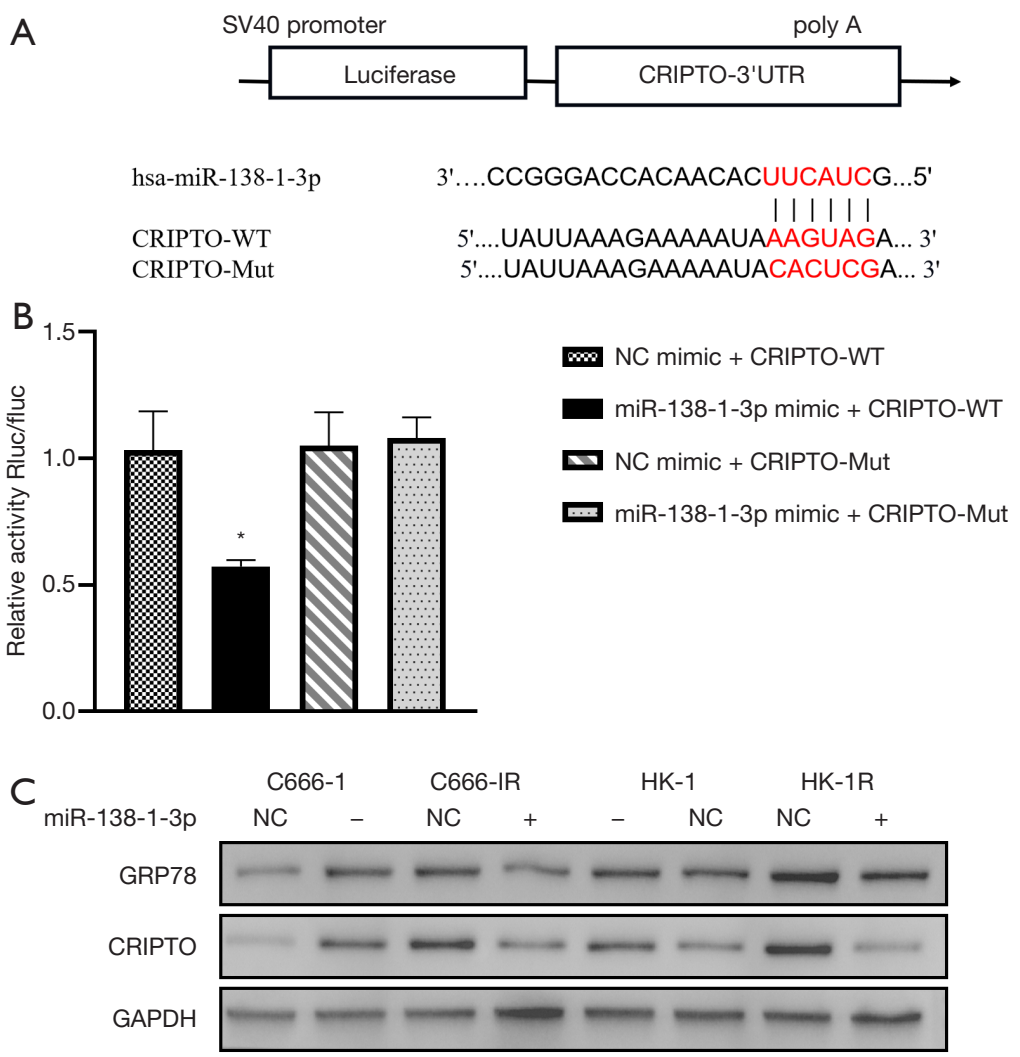

Figure 4 MiR-138-1-3p downregulates CRIPTO expression. (A) Schematic diagram of the binding site of miR-138-1-3p targeting the 3'UTR of CRIPTO. (B) Luciferase reporter gene detecting the luciferase activity of CRIPTO-WT and CRIPTO-MUT in different transfected HEK-293T cells. (C) The expression of CRIPTO and GRP78 in C666-1, C666-1R, HK-1, and HK-1R cells after altering miR138-1-3p levels. ${ }^{*} \mathrm{P}<0.05$, NC mimic + CRIPTO-WT vs. miR-138-1-3p mimic + CRIPTO-WT.

green-labeled CRIPTO signal (Figure 5D). Combined with the previous protein expression results, we speculate that miR-138-1-3p can inhibit the expression of CRIPTO, thereby affecting its binding to GRP78 on the cell membrane. Furthermore, miR-138-1-3p can affect the stem cell characteristics of tumor cells and increase their sensitivity to radiation.

\section{MiR-138-1-3p inbibits the FAK2/STAT3 pathway and increases the sensitivity of NPC cells to radiation}

It has been reported that the activation of the JAK2/STAT3 signaling pathway can change the invasion and metastasis capabilities of tumor cells (27). CRIPTO can enhance tumor cell proliferation and survival by participating in the JAK2/STAT3 pathway (18). We previously demonstrated that miR-138-1-3p can regulate CRIPTO. Thus, we attempted to verify whether miR-138-1-3p can modulate the JAK2/STAT3 signaling pathway. We observed that when miR-138-1-3p expression was upregulated, the expression levels of CRIPTO, JAK2, STAT3, p-JAK2, and p-STAT3 were all decreased (Figure 6A,B). Conversely, when miR-138-1-3p expression was downregulated, the expression levels of CRIPTO, JAK2, STAT3, p-JAK2, and p-STAT3 were all increased (Figure 6A,B). Cell proliferation experiments showed that the upregulation of miR-138-1$3 \mathrm{p}$ expression could inhibit the proliferation of NPC cells after 6 Gy irradiation (Figure $6 C, D$ ). These results indicate that miR-138-1-3p can inhibit the activation of the JAK2/ STAT3 signaling pathway by targeting CRIPTO, thereby inhibiting the proliferation of NPC cells.

\section{Discussion}

MiRNAs are present in almost all cellular pathways of human cancer (28) and play significant roles in the occurrence, development, migration, and metastasis of cancer. Global miRNA profiling from many patient cohorts 
A

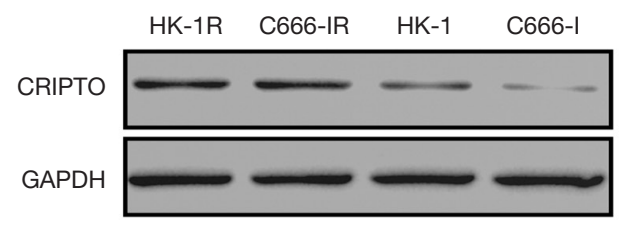

C
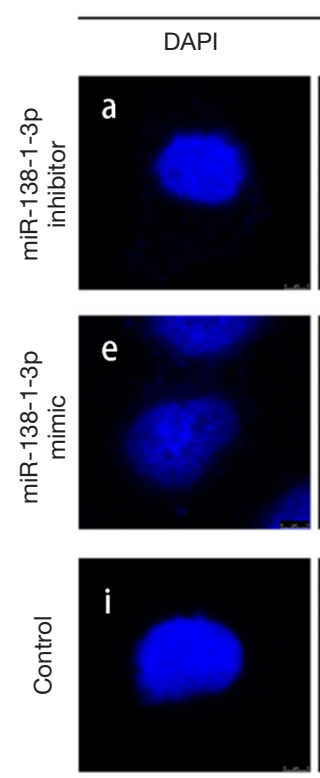

C666-IR
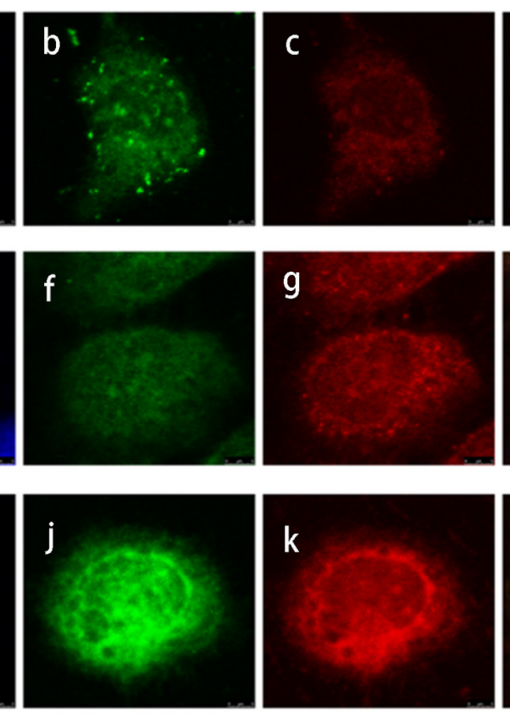

B

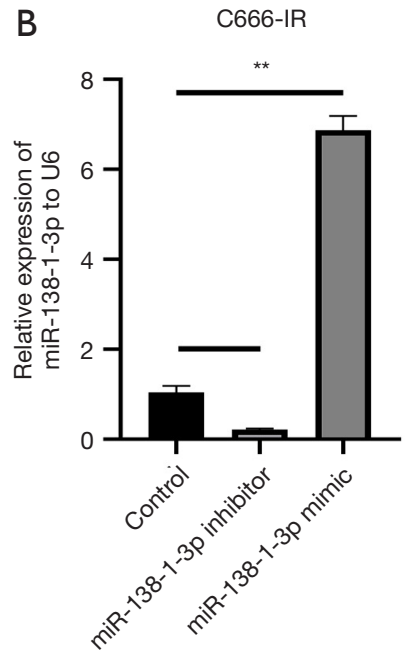

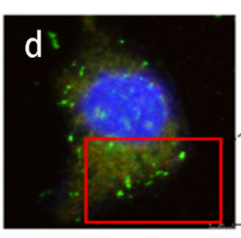
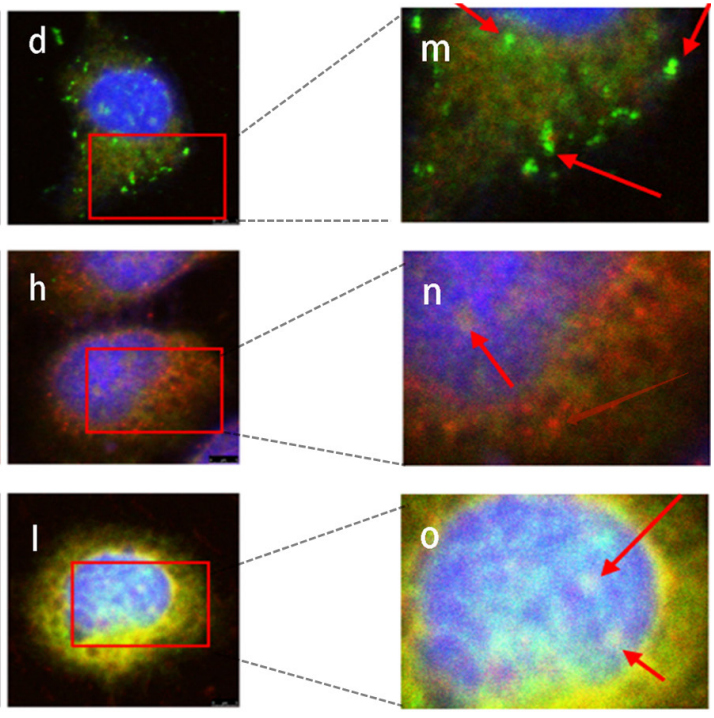

Figure 5 MiR-138-1-3p affects the stability of the CRIPTO-GRP78 complex in the cell membrane. (A) The expression of CRIPTO in HK1R, C666-1R, HK-1, and C666-1 cells by western blot. (B) The expression of miR-138-1-3p after C666-IR cells were transfected with miR138-1-3p inhibitor and miR-138-1-3p mimic. (C, D) Confocal fluorescence imaging of the localization of CRIPTO and GRP78 in C666-1R cells. Confocal fluorescence imaging of the localization of CRIPTO and GRP78 in C666-1R cells, (m), (n), (o) are the enlarged images of the fixed field of view of (d), (h), (l). The red arrow points to the complex formed by CRIPTO and GRP78. ${ }^{*} \mathrm{P}<0.05,{ }^{* *} \mathrm{P}<0.01$.

has identified miR-138 as a tumor suppressor marker in various types of cancer (29). In our study, using the chips of C666-1 and C666-1R NPC cells, we showed a significant reduction in miR-138-1-3p expression in radiationresistant cells. The trend was consistent in HK-1 and HK-1R NPC cells, as revealed by qRT-PCR. Subsequent experiments demonstrated that miR-138-1-3p is related to the radioresistance of NPC tumor cells, as the higher the expression of miR-138-1-3p, the more sensitive the NPC cells are to radiation. Next, we explored the mechanism by which miR-138-1-3p affects the radiosensitivity of NPC cells. We found that altering the miR-138-1-3p levels in HK- 1 and HK-1R cells also altered E-cadherin, $\mathrm{N}$-cadherin, and vimentin protein expression levels, as well as their 


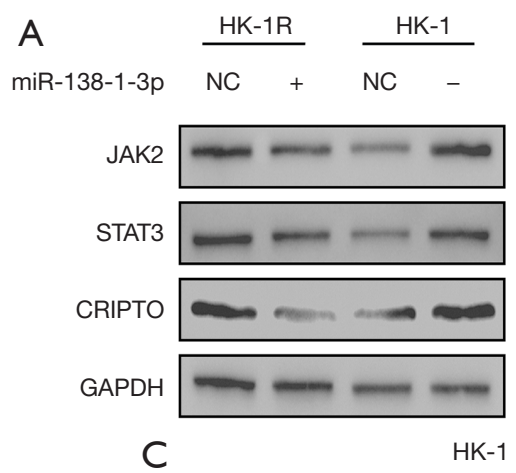

B

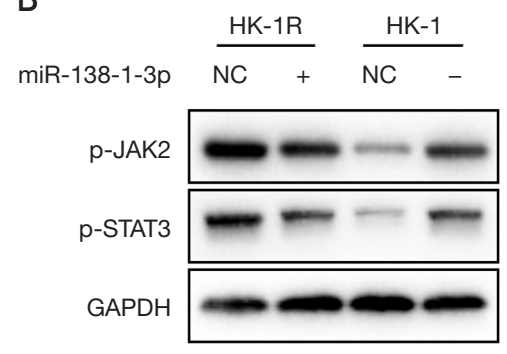

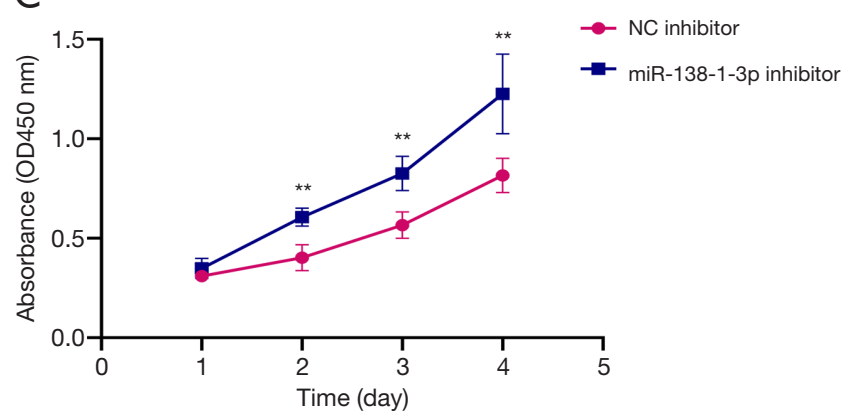

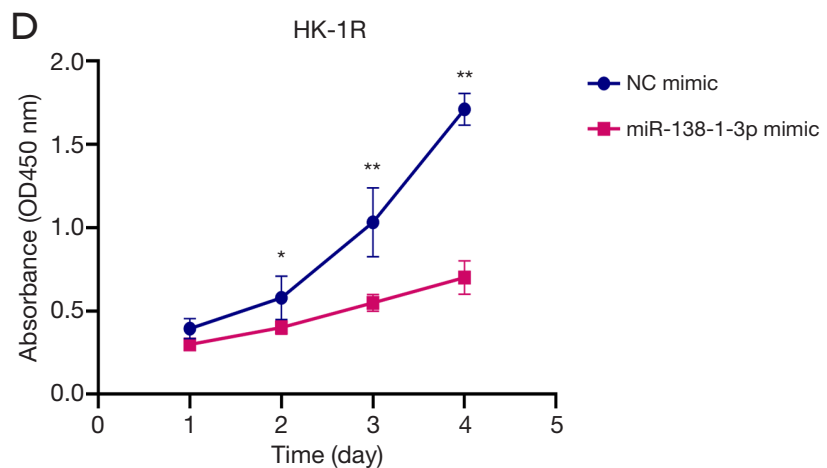

Figure 6 MiR-138-1-3p inhibits the JAK2/STAT3 signaling pathway. (A,B) The expressions of CRIPTO, JAK2, STAT3, p-JAK2, and p-STAT3 after altering miR-138-1-3p levels. (C) The viability of HK-1 cells transfected with NC inhibitor and miR-138-1-3p inhibitor after 6 Gy irradiation was detected by the CCK-8 assay. (D) The viability of HK-1R cells transfected with NC mimic and miR-138-1-3p mimic after 6 Gy irradiation was detected by the CCK-8 assay. ${ }^{*} \mathrm{P}<0.05,{ }^{* *} \mathrm{P}<0.01$.

migration abilities, which are suggestive of the correlation between miR-138-1-3p and the EMT pathway.

Previous reports have shown that activating the EMT pathway can render tumor cells resistant to radiotherapy (7). Therefore, the radiosensitization of NPC by miR-138$1-3 p$ can be attributed to its ability to negatively regulate the cell migration and EMT of tumor cells. MiR-138-1$3 p$ affects the EMT pathway, and a literature search yields CRIPTO as one of the key molecules in EMT (30). By using the Target Scan Human 7.2 database, we predicted that miR-138-1-3p targets CRIPTO as a downstream molecule, which was confirmed via the luciferase reporter assay and western blotting experiments, demonstrating that miR-138-1-3p can regulate the expression levels of CRIPTO. Several studies have shown that CRIPTO and GRP78 can form a complex on the cell membrane and activate the JAK2/STAT3 pathway, imparting stem cell like characteristics in the host cell with increased proliferation activity $(18,31)$. Our immunohistochemical analysis revealed the colocalization of CRIPTO and GRP78 on the cell membrane that changed when miR-138-1-3p expression was altered. Specifically, the expression of CRIPTO on the 
cell surface was inversely correlated to the miR-138-1-3p levels in NPC cells. Furthermore, western blotting revealed the changes in JAK2 and STAT3 levels in NPC cells in response to altered miR-138-1-3p levels, as speculated.

Collectively, these findings confirmed that miR-138-1$3 p$ can affect the activation of the JAK2/STAT3 signaling pathway by targeting CRIPTO, and can therefore affect the EMT pathway of NPC cells. Consequently, miR-138-1-3p alters the sensitivity of NPC cells to radiation, confirming the significance of miR-138-1-3p as a clinical target in NPC radiotherapy.

This study still has some limitations. Although these findings have demonstrated that miR-138-1-3p can regulate the radiosensitivity of NPCs under in vitro conditions, its in vivo relevance and effective application in clinical practice need validation in future studies. Furthermore, the mechanism by which CRIPTO activates the JAK2/STAT3 signaling pathway was not elucidated in the current study, and will therefore be investigated in our future research.

\section{Conclusions}

MiR-138-1-3p is downregulated in radioresistant NPC cells, which if upregulated, can inhibit the EMT pathway by targeting CRIPTO and the JAK2/STAT3 signaling pathway, consequently rendering NPC tumor cells sensitive to radiotherapy.

\section{Acknowledgments}

Funding: This study was supported by grants from the National Natural Science Foundation of China (81372905 to XF), Hunan Provincial Natural Science Foundation of China (2017JJ2384 to XL; 2020JJ8112 to XF), and Postgraduate Student Research and Innovation Projects of Central South University (2019zzts798 and 2019zzts1038).

\section{Footnote}

Reporting Checklist: The authors have completed the MDAR reporting checklist. Available at http://dx.doi.org/10.21037/ atm-21-521

Data Sharing Statement: Available at http://dx.doi. org/10.21037/atm-21-521

Conflicts of Interest: All authors have completed the ICMJE uniform disclosure form (available at http://dx.doi. org/10.21037/atm-21-521). The authors have no conflicts of interest to declare.

Ethical Statement: The authors are accountable for all aspects of the work in ensuring that questions related to the accuracy or integrity of any part of the work are appropriately investigated and resolved.

Open Access Statement: This is an Open Access article distributed in accordance with the Creative Commons Attribution-NonCommercial-NoDerivs 4.0 International License (CC BY-NC-ND 4.0), which permits the noncommercial replication and distribution of the article with the strict proviso that no changes or edits are made and the original work is properly cited (including links to both the formal publication through the relevant DOI and the license). See: https://creativecommons.org/licenses/by-nc-nd/4.0/.

\section{References}

1. Chen W, Hu GH. Biomarkers for enhancing the radiosensitivity of nasopharyngeal carcinoma. Cancer Biol Med 2015;12:23-32.

2. Feng X, Lv W, Wang S, et al. miR-495 enhances the efficacy of radiotherapy by targeting GRP78 to regulate EMT in nasopharyngeal carcinoma cells. Oncology Reports 2018;40:1223-32.

3. Qu C, Zhao Y, Feng G, et al. RPA3 is a potential marker of prognosis and radioresistance for nasopharyngeal carcinoma. J Cell Mol Med 2017;21:2872-83.

4. You R, Liu YP, Lin DC, et al. Clonal Mutations Activate the NF-kB Pathway to Promote Recurrence of Nasopharyngeal Carcinoma. Cancer Res 2019;79:5930-43.

5. Correia de Sousa M, Gjorgjieva M, Dolicka D, et al. Deciphering miRNAs' Action through miRNA Editing. Int J Mol Sci 2019;20:6249.

6. Wang $\mathrm{Y}$, Zhang Z, Wang H, et al. miR-138-1* regulates aflatoxin B1-induced malignant transformation of BEAS-2B cells by targeting PDK1. Arch Toxicol 2016;90:1239-49.

7. de Jong MC, Ten Hoeve JJ, Grénman R, et al. Pretreatment microRNA Expression Impacting on Epithelial-to-Mesenchymal Transition Predicts Intrinsic Radiosensitivity in Head and Neck Cancer Cell Lines and Patients. Clin Cancer Res 2015;21:5630-8.

8. Ma H, Yuan L, Li W, et al. The LncRNA H19/miR-193a$3 \mathrm{p}$ axis modifies the radio-resistance and chemotherapeutic tolerance of hepatocellular carcinoma cells by targeting PSEN1. J Cell Biochem 2018;119:8325-35. 
9. Jia J, Zhang X, Zhan D, et al. LncRNA H19 interacted with miR-130a-3p and miR-17-5p to modify radioresistance and chemo-sensitivity of cardiac carcinoma cells. Cancer Med 2019;8:1604-18.

10. Wang S, Claret FX, Wu W. MicroRNAs as Therapeutic Targets in Nasopharyngeal Carcinoma. Front Oncol 2019;9:756.

11. Tan LP, Tan GW, Sivanesan VM, et al. Systematic comparison of plasma EBV DNA, anti-EBV antibodies and miRNA levels for early detection and prognosis of nasopharyngeal carcinoma. Int J Cancer 2020;146:2336-47.

12. Sha HH, Wang DD, Chen D, et al. MiR-138: A promising therapeutic target for cancer. Tumour Biol 2017;39:1010428317697575.

13. Li J, Xia W, Su X, et al. Species-specific mutual regulation of p53 and miR-138 between human, rat and mouse. Sci Rep 2016;6:26187.

14. Minchiotti G, Parisi S, Liguori GL, et al. Role of the EGF-CFC gene cripto in cell differentiation and embryo development. Gene 2002;287:33-7.

15. Saloman DS, Bianco C, Ebert AD, et al. The EGF-CFC family: novel epidermal growth factor-related proteins in development and cancer. Endocr Relat Cancer 2000;7:199-226.

16. Minchiotti G, Manco G, Parisi S, et al. Structure-function analysis of the EGF-CFC family member Cripto identifies residues essential for nodal signalling. Development 2001;128:4501-10.

17. Shen MM, Schier AF. The EGF-CFC gene family in vertebrate development. Trends Genet 2000;16:303-9.

18. Yun S, Yun CW, Lee JH, et al. Cripto Enhances Proliferation and Survival of Mesenchymal Stem Cells by Up-Regulating JAK2/STAT3 Pathway in a GRP78Dependent Manner. Biomol Ther (Seoul) 2018;26:464-73.

19. Jin J, Guo Q, Xie J, et al. Combination of MEK Inhibitor and the JAK2-STAT3 Pathway Inhibition for the Therapy of Colon Cancer. Pathol Oncol Res 2019;25:769-75.

20. Zhang C, Liu J, Yuan C, et al. JAK2/STAT3 is associated with the inflammatory process in periapical granuloma. Int J Clin Exp Pathol 2019;12:190-7.

Cite this article as: Du T, Jiang J, Chen Y, Zhang N, Chen G, Wang X, Long X, Feng X. MiR-138-1-3p alters the stemness and radiosensitivity of tumor cells by targeting CRIPTO and the JAK2/STAT3 pathway in nasopharyngeal carcinoma. Ann Transl Med 2021;9(6):485. doi: 10.21037/atm-21-521
21. Xie C, Wu Y, Fei Z, et al. MicroRNA-1275 induces radiosensitization in oesophageal cancer by regulating epithelial-to-mesenchymal transition via $\mathrm{Wnt} /$-catenin pathway. J Cell Mol Med 2020;24:747-59.

22. Zhang P, Wei Y, Wang L, et al. ATM-mediated stabilization of ZEB1 promotes DNA damage response and radioresistance through CHK1. Nat Cell Biol 2014;16:864-75.

23. Mittal V. Epithelial Mesenchymal Transition in Tumor Metastasis. Annu Rev Pathol 2018;13:395-412.

24. Watanabe K, Meyer MJ, Strizzi L, et al. Cripto-1 is a cell surface marker for a tumorigenic, undifferentiated subpopulation in human embryonal carcinoma cells. Stem Cells 2010;28:1303-14.

25. Shani G, Fischer WH, Justice NJ, et al. GRP78 and Cripto form a complex at the cell surface and collaborate to inhibit transforming growth factor beta signaling and enhance cell growth. Mol Cell Biol 2008;28:666-77.

26. Wu MJ, Jan CI, Tsay YG, et al. Elimination of head and neck cancer initiating cells through targeting glucose regulated protein78 signaling. Mol Cancer 2010;9:283.

27. Liang JX, Gao W, Zeng XW, et al. SUMO4 small interfering RNA attenuates invasion and migration via the JAK2/STAT3 pathway in non-small cell lung cancer cells. Oncol Lett 2020;20:225.

28. Patil N, Allgayer H, Leupold JH. MicroRNAs in the Tumor Microenvironment. Adv Exp Med Biol 2020;1277:1-31.

29. Yeh M, Oh CS, Yoo JY, et al. Pivotal role of microRNA-138 in human cancers. Am J Cancer Res 2019;9:1118-26.

30. Chen L, Boleslaw Olszewski M, Kruithof-de Julio M, et al. Zebrafish Microenvironment Elevates EMT and CSC-Like Phenotype of Engrafted Prostate Cancer Cells. Cells 2020;9:797.

31. Kim S, Yoon YM, Han YS, et al. Administration of Cripto in GRP78 overexpressed human MSCs enhances stem cell viability and angiogenesis during human MSC transplantation therapy. Cell Prolif 2018;51:e12463. 